\title{
Can I Have the Real Doctor?
}

\author{
Elizabeth R. Steuber ${ }^{1}$ (i)
}

Received: 14 March 2021 / Accepted: 13 May 2021 / Published online: 24 May 2021

(C) Academic Psychiatry 2021

It was early into my intern year when I met Mr. J., a sixty-some year-old gentleman who had suffered a stroke immobilizing the left side of his body. I introduced myself as a doctor on the neurology team that would be caring for him during his hospitalization. Our introductory meeting was uneventful. Just as I neared the exit, however, Mr. J. asked to see my badge. I initially thought nothing of it since patients often forget my name in the hospital, and I returned to his bedside. As he read my badge, his brow furrowed. He sheepishly asked to see the badge a bit closer and then asked the actual question weighing on his mind: "Can I have the real doctor?"

At first, I was confused by Mr. J.'s request, even wondering whether this was a symptom of the neurologic insult he had endured. I again explained that I was a physician on the neurology team, taking care to put particular emphasis on the word "doctor." However, he glanced sideways and said again, "I know, you're wonderful, but I just want the real doctor."

It was at that moment I realized it was my badge that had come between us. As a psychiatry trainee, my hospital badge says both "Psychiatry" and "Doctor" below my name. I typically view this as a badge of honor, a hard-fought prize for years of studies and hard work. However, my badge was now seemingly working against me - despite the big, bolded letters spelling "Doctor," he viewed me as something less than a true physician.

I was taught by mentors throughout medical school that, as a woman in medicine, these types of interactions would occur. Patients would not view me as the physician, instead looking to my male counterparts for treatment and insight $[1,2]$. Interestingly, such an encounter had not yet occurred in my training. Instead, rather than my gender, my chosen specialty was the barrier to my success as a burgeoning young doctor.

Elizabeth R. Steuber esteube1@jhmi.edu

1 Johns Hopkins Hospital, Baltimore, MD, USA
Despite the scientific advancements in the field, the increased competitiveness, and the decreased stigmatization of mental health care, psychiatry still appears to be the ugly duckling of medicine. Psychiatric illnesses are still separated from "medical problems" throughout much of the world. As such, psychiatrists do not often achieve parity as true medical doctors, despite their training to the contrary [3]. Caricatures of armchair psychiatrists unable to interpret basic lab results or archaic notions of psychiatry as the study of maternal imperfections continue to permeate public perceptions of the field, despite tremendous reductions in bias against mental illness over the past decades. As a result, the negative stereotypes perpetuated in popular culture fundamentally alter how psychiatry, and therefore psychiatrists, is perceived [4].

Despite his request, what Mr. J. needed in that moment was a psychiatrist. His history revealed that he was desperately anxious about his trajectory and demoralized by his poststroke loss of function. This event was a devastating blow to his identity and his self-esteem. It also raised questions of how he would continue to care for his family and be independent. My psychiatric training taught me that my competence was not Mr. J.'s core concern. His question was a communication of fear. He did not need to hear about my qualifications, the medical school I graduated from, or my test scores; he needed to know that I cared and that I would fight for him.

I took a seat by Mr. J.'s bed and was honest with him. I told him the next several months to years would be challenging, but that it was my role to help him and his family through this event. I knew that for him, having an idea of what to expect and what he could do was critical to helping him feel some semblance of control in an uncontrollable situation. The limits of medicine did not allow me to cure Mr. J.'s stroke that afternoon. Instead, as his doctor, I sat with his emotion in the face of indescribable sadness and fear. We then worked together to create a path forward that was focused on what Mr. J. needed to feel empowered. In turn, Mr. J. looked at me as "the real doctor," a person he could turn to for guidance in his darkest hour. 
All psychiatry trainees complete several months on general medicine and neurology services, caring for patients, like Mr. J., in the role of the "medical" doctor on the primary team. At a time when isolation is the norm and family members are now just voices through a telephone, an emphasis on the psychosocial drivers of well-being, in combination with human physiology, is critical for treating disease. In this brave new world, perhaps more than ever, psychiatrists are the medical doctors most needed in the hospital to facilitate the care of patients. I am hopeful that by leading through example on the medical floors, psychiatry trainees will continue to reshape how the field is seen by society at-large, even if it is only one patient at a time. Such perceptual shifts are critical for breaking down the remaining stigma against psychiatry that continues to limit the field's reach.

Mr. J. left the hospital several days later. As he left, he called out to me in the hall, "Hey Doc, you're not so bad... for a shrink." He then threw me one of his characteristic winks, and I grinned behind my mask. We both knew he'd gotten good care from a real doctor.

\section{Declarations}

Disclosures The author states that there is no conflict of interest.

\section{References}

1. Cao J, Banaji MR. The base rate principle and the fairness principle in social judgment. Proc Natl Acad Sci. 2016;113(27):7475-80.

2. Cronauer CK, Mast MS. Hostile sexist male patients and female doctors: a challenging encounter. Patient. 2014;7:37-45.

3. Gaebel W, Zäske H, Zielasek J, Cleveland HR, Samjeske K, Stuart $\mathrm{H}$, et al. Stigmatization of psychiatrists and general practitioners: results of an international survey. Eur Arch Psychiatry Clin Neurosci. 2015;265(3):189-97.

4. Bhugra D, Sartorius N, Fiorillo A, Evans-Lacko S, Ventriglio A, Hermans MHM, et al. EPA guidance on how to improve the image of psychiatry and of the psychiatrist. Eur Psychiatry. 2015;30(3): 423-30.

Publisher's Note Springer Nature remains neutral with regard to jurisdictional claims in published maps and institutional affiliations. 